\title{
Parameter Research of UAV Vertical Rope-type Recovery System
}

\author{
Chengjing $\mathrm{Ma}^{1}$, Xing'an $\mathrm{Liu}^{2, \mathrm{a}}$ and Caiwen Zhang ${ }^{3}$ \\ ${ }^{1}$ College of Mechanical and Electrical Engineering, Nanjing University of Aeronautics and Astronautics, Nanjing \\ 210016, China \\ ${ }^{2}$ School of Aeronautic Science and Engineering, Beihang University, Beijing 100191, China \\ ${ }^{3}$ Research Institute of Unmanned Aerial Vehicle, Nanjing University of Aeronautics and Astronautics, Nanjing \\ 210016, China
}

\begin{abstract}
Vertical rope-type recovery system is a new accurate recovery method for small fixed-wing unmanned aerial vehicles (UAV). This paper models the recovery system and finishes the simulation of recovery process. The recovery stand model of the recovery system is built by analyzing the physical essence, and the aircraft model is based on dynamics and kinematics equations as well as the mechanical characteristics of arresting rope. Finally the simulation analysis of the recovery process is completed. On the basis of the modeling simulation, the system parameters' effect on recovery performance such as maximum resistance force, maximum overload and maximum radius has been discussed by varying each key parameter. Eventually, the pattern of how initial conditions when hitting the rope as well as the arresting rope's stiffness and damping coefficient influence recovery performance has been researched, and the result could provide theoretical reference for the design of UAV using vertical rope-type recovery and the system in the future.
\end{abstract}

\section{Introduction}

The development of UAV technology has been mature so far, and it could undertake a large range of tasks [1]. Because of its convenient operation, flexible deployment and excellent performance-cost ratio, the deployment area has been extended to ships, islands and other locations [2]. Vertical ropetype recovery is a way to achieve accurate recovery in these limited areas. It's especially favourable for small fixed-wing UAV, due to the large recovery window during the recycle process, low demand for control accuracy in the height direction, and low cost in terms of mass and volume [3]. The US military has successfully developed UAV vertical rope-type recovery system and deployed it, for instance, the Scan Eagle UAV and RQ-21 UAV, built by INSITU corporation, have used sky-hook recovery system $[4,5]$.

So far, many research institutes and schools have explored this technology. For example, Dai Chuan et al. of Chengdu Aircraft Design Institute proposed a vertical rope-type recovery device of UAV [6]; Lu Wei from Northwestern Polytechnical University simulated the UAV dynamic characteristics, buffer and energy-absorbing capability during the rope-hook recovery process, and researched the feasibility of rope-hook recovery mode [7]. However, the recovery stand model in

\footnotetext{
a Xing'an Liu : liuxingan94@163.com
} 
current research differs from the actual recycling devices in use, and the influence pattern of the system parameters on the recovery performance is not studied in depth.

This paper improves the recovery system model based on the actual physical characteristics of the vertical rope-type recovery system, and uses it for simulation. On the basis of it, the influence of initial conditions when hitting the rope and the arresting rope's stiffness and damping coefficient on recovery performance has been researched, and it could provide reference for design of UAV and recovery system.

\section{Recovery system modelling}

Analysis of the recycling device shows that the vertical rope-type recovery system is mainly composed of a ground recovery stand, arresting rope device and the intercepting hook on wing tip [8]. The recovery system can be simplified as Figure 1. In the figure, the fixed point of the recovery system on the ground is point $\mathrm{Q}, \mathrm{QH}$ is the vertical bar, QB and AD are cross bars, composing the main body of the recovery stand. $\mathrm{AD}$ could rotate around $\mathrm{QH}$, and is constrained by two damping rope $\mathrm{EF}$ and FG. The arresting rope is represented by $\mathrm{PAB}$ in the figure, the damper which is simplified as the stiffness and damping coefficient of arresting rope in subsequent research. As for the aircraft, the center of gravity is point $\mathrm{K}$, the point of wing root is point $\mathrm{I}$, the point of wing tip is point $\mathrm{J}$, and the point which the leading edge of UAV wing contacts the arresting rope is point $\mathrm{C}$.

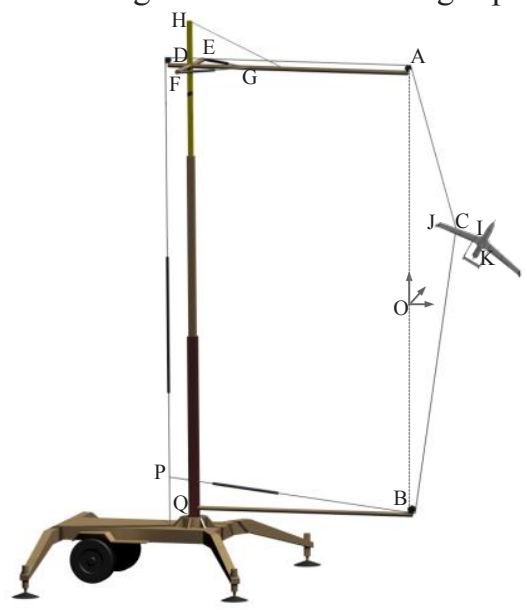

Figure 1. Schematic diagram of vertical rope-type recovery system

\subsection{Recovery stand modelling}

The recovery stand will deform due to the force transferred by the arresting rope during the recovery, and the process of its deformation and recovery plays a role of buffer and energy absorption. Therefore, it can't be considered as rigid body during simulation. According to the actual dynamic and kinetic characteristics of the recovery stand, it can be simplified to two parts which are connected together. Among them, bracket $\mathrm{QH}$ is fixed on the ground, therefore only the bending and torsion deformation is considered. Strut $\mathrm{AD}$ is hinged on bracket $\mathrm{QH}$, and could only rotate around the $\mathrm{QH}$ axis. Hence, during the dynamic analysis, the recovery stand is assumed balanced by overall external forces, and only the change of attitude angle caused by the external moment is considered, so the dynamic equation is:

$$
M_{S}=\boldsymbol{I}_{S} \boldsymbol{\omega}_{S}+\dot{\boldsymbol{\omega}}_{S} \times\left(\boldsymbol{I}_{S} \boldsymbol{\omega}_{S}\right)
$$

In the formula, $\omega_{S}$ is the angular velocity of the recovery stand in the local coordinate system, $\boldsymbol{I}_{S}$ is the inertia tensor of the recovery stand. 
For strut $\mathrm{AD}$, there are 3 external forces on it, the reaction force $\boldsymbol{F}_{C A}$ of the arresting rope, the resistance force $\boldsymbol{F}_{F G}$ and $\boldsymbol{F}_{E G}$ caused by damping rope, as shown in Figure 2. Its resultant force equation is not considered as the assumption that the force is balanced, so these 3 forces only cause it rotating around axis $\mathrm{QH}$.

The moment for the whole recovery stand caused by $\boldsymbol{F}_{C A}$ can be calculated as:

$$
\boldsymbol{M}_{C A}=\boldsymbol{r}_{C A} \times \boldsymbol{F}_{C A}
$$

The component of moment $\boldsymbol{M}_{C A}$ in plane ADF causes strut AD rotating around axis $\mathrm{QH}$, the other components transmit to strut $\mathrm{QH}$ through the hinge, and are finally supported by $\mathrm{QH}$.

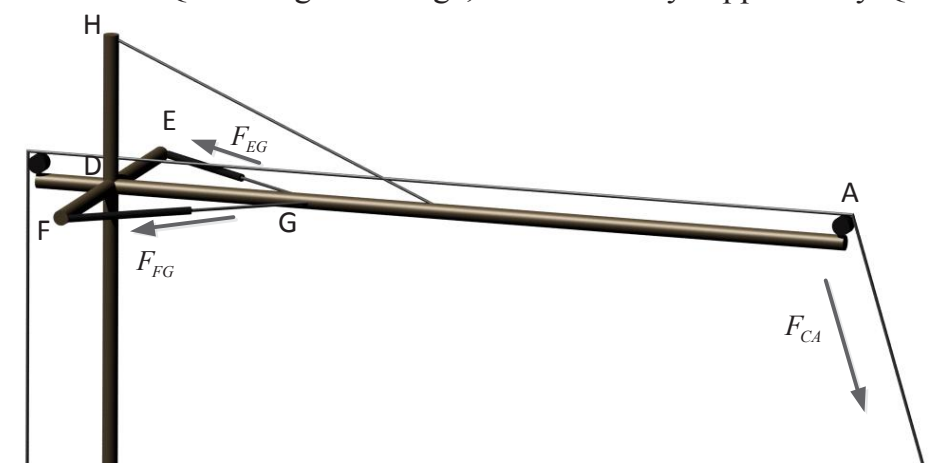

Figure 2. Force analysis of strut $A D$

The resistance force $\boldsymbol{F}_{F G}$ and $\boldsymbol{F}_{E G}$ caused by damping rope can be calculated as:

$$
F=k \Delta l+c \Delta l
$$

In the formula, $k$ and $c$ denote stiffness and damping coefficient of the damping rope respectively. The change of length $\Delta l$ and the rate of the change $\Delta l$ are related to the position change of point $\mathrm{E}, \mathrm{F}$ and $\mathrm{G}$, which can be obtained by the coordinate of these points. The resultant moment caused by the resistance force can be calculated as:

$$
\boldsymbol{M}_{E F}=\boldsymbol{r}_{E G} \times \boldsymbol{F}_{E G}+\boldsymbol{r}_{F G} \times \boldsymbol{F}_{F G}
$$

As for strut $\mathrm{QH}$, the external moments it takes are the resulting moment caused by the counter force of $\boldsymbol{F}_{F G}$ and $\boldsymbol{F}_{E G}$, and the moment generated by the force transmitted by the hinge point connected with strut AD. Its own material stiffness and damping are simplified into elastic stiffness and damping coefficient in the direction of three altitude angle, which can provide recovery moment and damping moment.

From the foregoing analysis, the position and rate of change of the position of the feature points on recovery stand need to be determined in the modeling process, which can be solved by determining the coordinates of these points in the same coordinate system. This paper uses the vertical ground coordinate system to determine the coordinates, and establishes local coordinate system to assist the determination. Set the origin of vertical ground coordinate system $V$ as point $\mathrm{O}$, and establish local coordinate system respectively with point $\mathrm{Q}$ and point $\mathrm{D}$ as the origin. So $\boldsymbol{r}_{O A}$ and $\dot{\boldsymbol{r}}_{O A}$ can be expressed as follow [9]:

$$
\begin{gathered}
\boldsymbol{r}_{O A}=\boldsymbol{r}_{O D}^{V}+\boldsymbol{T}_{V D} \cdot \boldsymbol{r}_{D A}^{D}=\boldsymbol{r}_{O Q}^{V}+\boldsymbol{T}_{V Q} \cdot \boldsymbol{r}_{Q D}^{Q}+\boldsymbol{T}_{V D} \cdot \boldsymbol{r}_{D A}^{D} \\
\dot{\boldsymbol{r}}_{O A}=\dot{\boldsymbol{r}}_{O Q}^{V}+\dot{\boldsymbol{T}}_{V Q} \cdot \boldsymbol{r}_{Q D}^{Q}+\boldsymbol{T}_{V Q} \cdot \dot{\boldsymbol{r}}_{Q D}^{Q}+\dot{\boldsymbol{T}}_{V D} \cdot \boldsymbol{r}_{D A}^{D}+\boldsymbol{T}_{V D} \cdot \dot{\boldsymbol{r}}_{D A}^{D}=\boldsymbol{\omega}_{Q}^{V} \times \boldsymbol{T}_{V Q} \cdot \boldsymbol{r}_{Q D}^{Q}+\boldsymbol{\omega}_{D}^{V} \times \boldsymbol{T}_{V D} \cdot \boldsymbol{r}_{D A}^{D} \\
=\boldsymbol{\omega}_{Q}^{V} \cdot \boldsymbol{r}_{Q D}^{V}+\boldsymbol{\omega}_{D}^{V} \cdot \boldsymbol{r}_{D A}^{V}
\end{gathered}
$$

In the formula, $\boldsymbol{r}_{O D}^{V}$ means the vector $\boldsymbol{r}_{O D}$ expressed in vertical ground coordinate system $V, \boldsymbol{r}_{D A}^{D}$ means the vector $\boldsymbol{r}_{D A}$ expressed in the local coordinate system with point $\mathrm{D}$ as the origin, $\boldsymbol{T}_{V D}$ represents the transformation matrix from local coordinate system $D$ to vertical ground coordinate system $V$. Other similar expressions in this paper have the same meaning, and if no special reference 
is made, the default coordinate system is vertical ground coordinate system $V . \boldsymbol{r}_{O G}, \dot{\boldsymbol{r}}_{O G}, \boldsymbol{r}_{O E}, \dot{\boldsymbol{r}}_{O E}$, $\boldsymbol{r}_{O F}$ and $\dot{\boldsymbol{r}}_{O F}$ can also be obtained using similar method in appropriate coordinate system. Then we can use the results to get relevant forces and moments, and the recovery stand can be modeled based on the dynamics analysis above.

\subsection{Aircraft modelling}

The aircraft six-degree-of-freedom dynamic equations are as follow [10]:

$$
\left\{\begin{array} { l } 
{ X - m g \operatorname { s i n } \theta = m ( \dot { u } + q w - r v ) } \\
{ Y + m g \operatorname { c o s } \theta \operatorname { s i n } \phi = m ( \dot { v } + r u - p w ) } \\
{ Z + m g \operatorname { c o s } \theta \operatorname { c o s } \phi = m ( \dot { w } + p v - q u ) }
\end{array} \left\{\begin{array}{l}
L=I_{x} \dot{p}-I_{z x}(\dot{r}+p q)-\left(I_{y}-I_{z}\right) q r \\
M=I_{y} \dot{q}-I_{z x}\left(r^{2}-p^{2}\right)-\left(I_{z}-I_{x}\right) r p \\
N=I_{z} \dot{r}-I_{z x}(\dot{p}-q r)-\left(I_{x}-I_{y}\right) p q
\end{array}\right.\right.
$$

Compared with conventional aircrafts, additional resistance force and resulting moment are added due to the arresting rope during the vertical rope-type recovery. Therefore, the modelling of the aircraft needs to increase the force $\boldsymbol{F}_{l}$ and moment $\boldsymbol{M}_{l}$ caused by the arresting rope on the basis of the dynamic equations of the aircraft.

Each force caused by the arresting rope $\mathrm{PAC}$ and $\mathrm{PBC}$ is along its own deformation direction, and the magnitude is as follows:

$$
F_{A C}=-\left(k_{l} \Delta l_{P A C}+c_{l} \Delta i_{P A C}\right), \quad F_{B C}=-\left(k_{l} \Delta l_{P B C}+c_{l} \Delta l_{P B C}\right)
$$

The resistance force caused by the arresting rope is the resultant force of $\boldsymbol{F}_{A C}$ and $\boldsymbol{F}_{B C}$ :

$$
\boldsymbol{F}_{l}=\boldsymbol{F}_{A C}+\boldsymbol{F}_{B C}
$$

In order to calculate the force, the recovery process is divided into two phases: in the first phase, the arresting rope hit the wing leading edge, and slide along the leading edge until the intercepting hook at wing tip; in the second phase, the arresting rope resistance effects on wing tip. Therefore, in view of the different physical characteristics of the two stages, the resistance of the arresting rope at each stage should be considered separately.

In the first stage, it can be assumed that the leading edge of the wing is smooth and there is no friction on the arresting rope, thus the rope is balanced itself and the constraint condition is:

$$
F_{A C}=F_{B C}
$$

Besides, we can consider the arresting rope as entirety because the rope can slide around the leading edge, which means:

$$
\dot{r}_{A C}+\dot{r}_{B C}={ }_{\Delta} \dot{l}_{P A C}+\dot{l}_{P B C}
$$

According to the geometric relationship, we got:

$$
\Delta l_{P A C}=\left|\boldsymbol{r}_{A C}\right|-h_{A}, \Delta l_{P B C}=\left|\boldsymbol{r}_{B C}\right|-h_{B}
$$

$h_{A}$ and $h_{B}$ respectively means the height between the initial hit-rope position and point A or point $\mathrm{B}$. Thus, in order to solve the constraint equations (10) and (11), we need to get $r_{A C}, r_{B C}, \dot{r}_{A C}$ and $\dot{r}_{B C}$, which can be determined by the coordinates of end point:

$$
\begin{gathered}
\boldsymbol{r}_{A C}=\boldsymbol{r}_{O C}-\boldsymbol{r}_{O A} \\
\dot{r}_{A C}=\dot{\boldsymbol{r}}_{A C} \cdot \frac{\boldsymbol{r}_{A C}}{\left|\boldsymbol{r}_{A C}\right|}=\left(\dot{\boldsymbol{r}}_{O C}-\dot{\boldsymbol{r}}_{O A}\right) \cdot \frac{\boldsymbol{r}_{A C}}{\left|\boldsymbol{r}_{A C}\right|}
\end{gathered}
$$

Because of the initial pre-tightening force, the position of the contact point $\mathrm{C}$ should satisfy the condition that it's on line IJ, the leading edge of the wing, and it minimizes the change of the length of the arresting rope (the distance to $\mathrm{AB}$ is the shortest). So point $\mathrm{C}$ is the intersection of the common perpendicular of line IJ and $\mathrm{AB}$ and line IJ. Solve the equations of common perpendicular and line IJ, we can get point C's coordinate $\boldsymbol{r}_{O C}=\left(x_{C}, y_{C}, z_{C}\right)$. 
In order to get $\dot{r}_{O C}$, Body Axis system with the centre of gravity point $\mathrm{K}$ as origin is established, and the coordinate of point $\mathrm{C}$ in Body Axis system can be expressed as: $\boldsymbol{r}_{K C}^{K}=\left[l_{I K}-l_{C} \tan \Lambda,-\left(b / 2-l_{C}\right), 0\right]^{T}$, in which $l_{I K}$ is the distance between wing root point I and point $\mathrm{K}$ in the direction of body, $l_{C}$ is the distance between point $\mathrm{C}$ and wing tip in the direction of wingspan, $\mathrm{b}$ is length of the wingspan, and $\Lambda$ is the sweep angle of wing leading edge.

Point C's coordinate in vertical ground ordinated system can be expressed as $\boldsymbol{r}_{O C}^{V}=\boldsymbol{r}_{O K}^{V}+\boldsymbol{T}_{V K} \cdot \boldsymbol{r}_{K C}^{K}$, and take the derivative with respect to time, we can get:

$$
\dot{\boldsymbol{r}}_{O C}^{V}=\dot{\boldsymbol{r}}_{O K}^{V}+\dot{\boldsymbol{T}}_{V K} \cdot \boldsymbol{r}_{K C}^{K}+\boldsymbol{T}_{V K} \cdot \dot{\boldsymbol{r}}_{K C}^{K}=\dot{\boldsymbol{r}}_{O K}^{V}+\boldsymbol{\omega}_{K}^{V} \times \boldsymbol{T}_{V K} \cdot \boldsymbol{r}_{K C}^{K}+\boldsymbol{T}_{V K} \cdot \dot{\boldsymbol{r}}_{K C}^{K}=\dot{\boldsymbol{r}}_{O K}^{V}+\boldsymbol{\omega}_{K}^{V} \times \boldsymbol{r}_{K C}^{V}+\boldsymbol{T}_{V K} \cdot \dot{\boldsymbol{r}}_{K C}^{K}
$$

$\boldsymbol{\omega}_{K}^{V}=[p, q, r]^{T}$ means the angular velocity vector of UAV in vertical ground coordinate system.

From the former modelling, $\boldsymbol{r}_{O A}, \boldsymbol{r}_{O B}$ and $\dot{\boldsymbol{r}}_{O A}, \dot{\boldsymbol{r}}_{O B}$ have been obtained, and therefore we can get $\boldsymbol{F}_{A C}$ and $\boldsymbol{F}_{B C}$, and finally we can get $\boldsymbol{F}_{l}$ and its resultant moment $\boldsymbol{M}_{l}=\boldsymbol{r}_{K C} \times \boldsymbol{F}_{l}$.

In the second stage, when the arresting rope gets locked by the intercepting hook, it couldn't slide in the direction of rope due to the friction between the hook and itself. Therefore the magnitude of two arresting rope's resistance force differs from each other and should be considered respectively. At this moment, point $\mathrm{C}$ coincides with point $\mathrm{J}$, and the constraint condition becomes as follow:

$$
\dot{r}_{A J}={ }_{\Delta} \dot{l}_{P A C}, \quad \dot{r}_{B J}={ }_{\Delta} \dot{l}_{P B C}
$$

Similar to the first stage, we should determine the coordinate of end points. The wing tip point J's coordinate in Body Axis system can be expressed as $\boldsymbol{r}_{K J}^{K}=\left[l_{I K}-b / 2 \tan \Lambda,-b / 2,0\right]^{T}$. Point J's coordinate in vertical ground ordinated system can be expressed as $\boldsymbol{r}_{O J}^{V}=\boldsymbol{r}_{O K}^{V}+\boldsymbol{T}_{V K} \bullet \boldsymbol{r}_{K J}^{K}$, and take the derivative with respect to time, we can get:

$$
\dot{\boldsymbol{r}}_{O J}^{V}=\dot{\boldsymbol{r}}_{O K}^{V}+\boldsymbol{\omega}_{K}^{V} \times \boldsymbol{r}_{K J}^{V}\left(\dot{\boldsymbol{r}}_{K J}^{B}=0\right)
$$

In the same way, we can get $\boldsymbol{F}_{l}$, using $\boldsymbol{r}_{O A}, \boldsymbol{r}_{O B}$ and $\dot{\boldsymbol{r}}_{O A}, \dot{\boldsymbol{r}}_{O B}$, and its resultant moment $\boldsymbol{M}_{l}=\boldsymbol{r}_{K C} \times \boldsymbol{F}_{l}$.

So far, the resistance force and moment in the recovery process can be determined. Combined with six-degree-of-freedom dynamic equations of conventional aircraft, the aircraft modelling is completed.

\section{Simulation of recovery process}

\subsection{Initial conditions}

Taking a small fixed-wing UAV as an example, we simulated the recovery process in the environment of Matlab/Simulink [11]. The UAV's parameters are as follow: the weight of UAV is $m=60 \mathrm{~kg}$, the length of wingspan is $b=5 \mathrm{~m}$, the leading edge sweep angle is $\Lambda=11^{\circ}$. The initial kinematics conditions are as follow: the vertical distance between the rope-contacting point and the upper end of the recovery stand point A and the vertical distance to point B is $h_{A}=h_{B}=8 \mathrm{~m}$, the distance between the rope-contacting point and wing tip in the direction of wingspan is $l_{C}=0.5 \mathrm{~m}$. The starting time of the simulation is when UAV hits the rope.

\subsection{Motion trajectory and change of velocity}

Figure 3 shows the motion trajectory of the center of gravity of the aircraft in horizontal plane. It shows that when the arresting rope slides along the leading edge of the wing at first, the attitude of the aircraft is almost unchanged and the original speed direction is maintained. After that, the arresting rope hangs on the wingtip. When the arresting rope was stretched long enough to provide centripetal 
force $\left(x_{K}=10 \mathrm{~m}\right.$ in the figure), the UAV enters the circular motion under the centripetal force provided by the resistance of the arresting rope, and the motion radius reduces continuously due to the energy absorption effect of recovery system. After about 2 laps, the aircraft speed decreased to the point where the circular motion could not be maintained, and then swung slightly around the equilibrium position until the speed was reduced to $2.5 \mathrm{~m} / \mathrm{s}$ below, which is thought the end of the recovery process.

The trajectory of the rope-contacting point in the horizontal plane is shown in Figure 4. It can be seen that when the arresting rope slides along the leading edge toward the wingtip, the motion trajectory of the rope-contacting point can be approximated as a straight line (as indicated by the dotted red line); then the trajectory of the rope-contacting point coincides with the wingtip trajectory, forming the fluctuation on the basis of the circular motion of aircraft as shown in the figure.

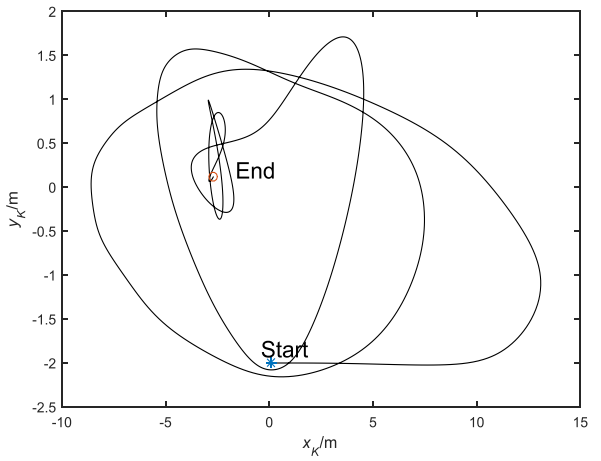

Figure 3. Trajectory of UAV's center of gravity in horizontal plane

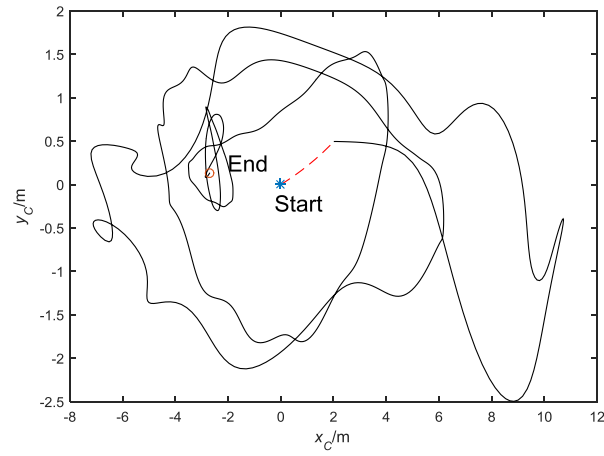

Figure 4. Trajectory of rope-contacting point in horizontal plane

The response curve of the UAV velocity $V_{a}$ with time $t$ in the recovery process is shown in Figure 5. It can be seen that the velocity of UAV dropped rapidly to less than $5 \mathrm{~m} / \mathrm{s}$ after two fluctuations within $5 \mathrm{~s}$, and then stabilized to below $2.5 \mathrm{~m} / \mathrm{s}$ after about $10 \mathrm{~s}$, when the UAV can be took down and recovered. Due to the energy absorption effect of the recovery stand, the peak of each fluctuation declines continuously.

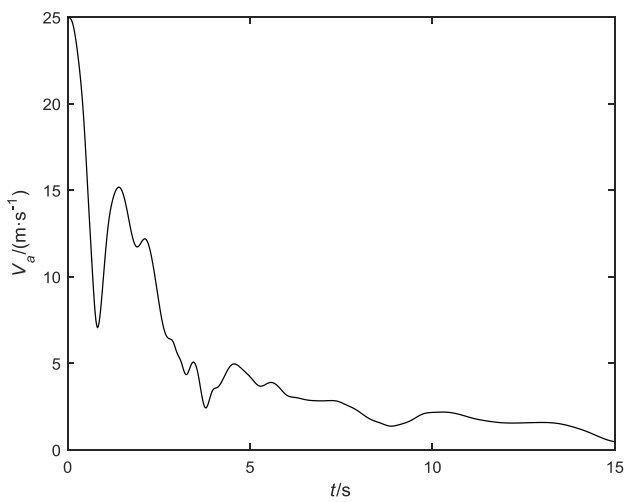

Figure 5. Curve of velocity of UAV during recovery

\subsection{Characteristics of resistance force}

When the parameters of arresting rope are set as $k_{l}=500 \mathrm{~N} / \mathrm{m}, c_{l}=40 \mathrm{~N} /\left(\mathrm{m} \cdot \mathrm{s}^{-1}\right)$, the curve of magnitude of resistance force $\left[F_{x}, F_{y}, F_{z}\right]$ and the resultant force $F_{R}$ is as Figure 6: 


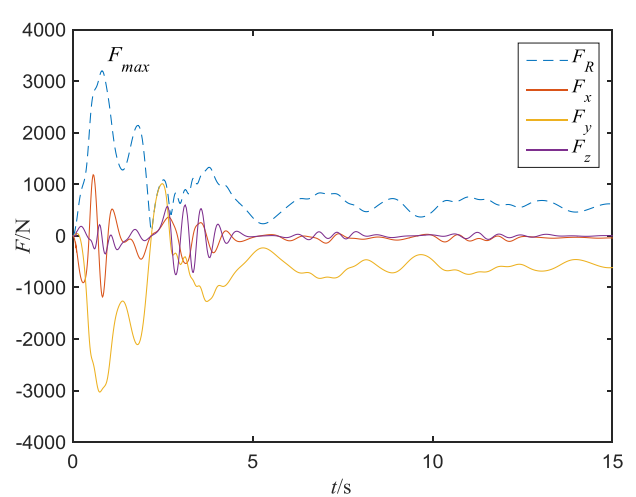

Figure 6. Curve of resistance force

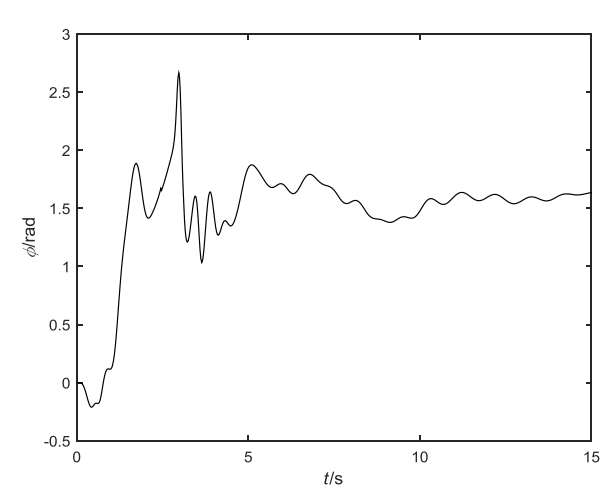

Figure 7. Curve of roll angle

It shows that the resistance force of arresting rope increases rapidly after the aircraft hit the rope and reaches a peak at about $0.8 \mathrm{~s}$, and the overload is about 5.41. At this moment, the aircraft moves to the farthest end from the balance position and begins to enter the circular motion. As for the change tendency of the resistance force in all directions, it shows that the force in the direction of fuselage (xaxis of the Body Axis) increases rapidly at first; however, in order to balance the centrifugal force of the aircraft after entering the circular motion, the force in the direction of wingspan(y-axis of the Body Axis) rapidly increases and become the main part of the total resistance; at last, when the aircraft reaches equilibrium, the arresting rope resistance almost exists only in the direction of wingspan, and is approximately equal to gravity, which is because at the equilibrium stage, the fuselage has a roll angle of about $90^{\circ}$ relative to the initial state (as shown in Figure 7), and the force of the arresting rope is in the direction of wingspan to balance gravity.

\section{Research on system parameters' effect on recovery performance}

\subsection{Effect of initial conditions when hitting the rope}

For a UAV with a definite appearance, the initial recovery velocity and the hit-rope position are uncertain factors and difficult to achieve accurate control. Therefore, the influence of the above factors on the recovery performance needs to be analysed.

During the recovery process, the initial recovery velocity of UAV determines the maximum kinetic energy, and thus affects the maximum resistance and maximum overload. But limited by maximum lift coefficient and the effect of the wind field, the recovery speed is usually higher than the stall speed. The influence of the recovery velocity $V_{0}$ on the maximum overload $n_{\max }$ is analysed under the setting of the system parameters of $k_{l}=500 \mathrm{~N} / \mathrm{m}, c_{l}=40 \mathrm{~N} /\left(\mathrm{m} \cdot \mathrm{s}^{-1}\right)$, the simulation result is as Figure 8:

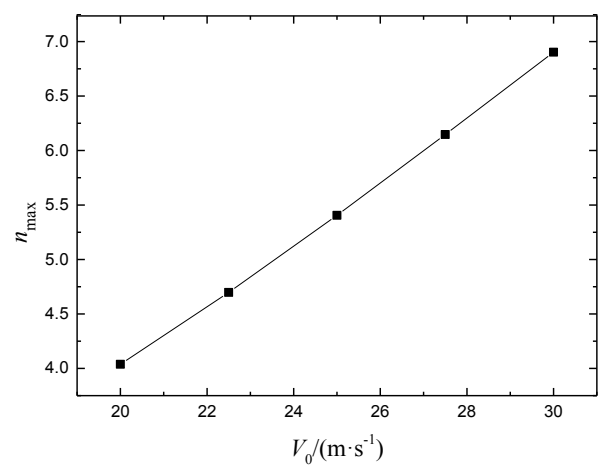

Figure 8. Influence of recovery velocity on maximum overload 
The figure shows that the effect of recovery velocity on the maximum overload is almost linear. It can be obtained that the maximum recovery speed should not exceed $33 \mathrm{~m} / \mathrm{s}$ through fitting, in order to ensure the maximum overload lower than 10.

The hit-rope position may vary in both the wingspan direction and the height direction. Therefore, how it impacts the recovery performance need to be analysed. Maintain the same parameters of arresting ropes, analysing the impact of the location of the arresting ropes on the maximum resistance $F_{\max }$ are shown in Figure 9 and Figure 10:

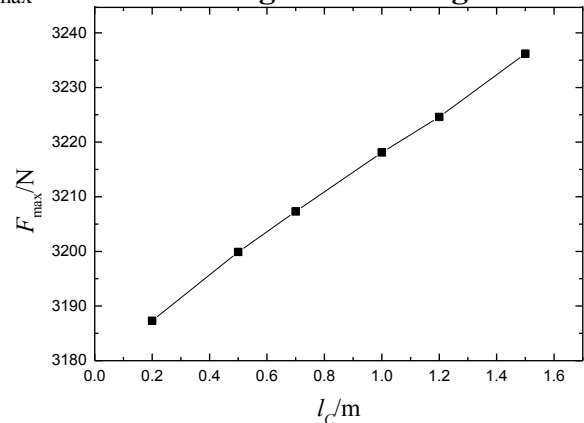

Figure 9. Influence of the hit-rope position along wingspan on maximum resistance force

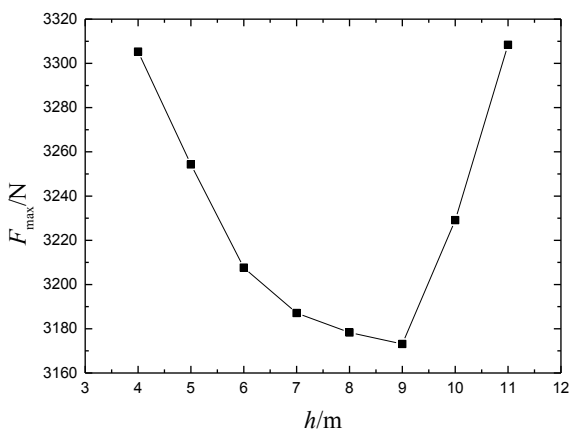

Figure 10. Influence of the height of the hit-rope position on maximum resistance force

It can be seen from the figure that the influence of the position change along the wingspan direction on the maximum resistance is monotonous. The closer the hit-rope position to wing tip is, the smaller the maximum resistance is. However, due to the short span of the wing, the range of maximum resistance is limited. Influence of the height change is non-monotonic. The resistance is the minimum when it's near the midpoint of the recovery stand. When the height of the hit-rope position is above the midpoint, the resistance is more sensitive to altitude changes.

\subsection{Effect of the stiffness and damping coefficient of arresting rope}

The recovery stand's stiffness and damping coefficient are related to its material, and are inconvenient to change, while the stiffness and damping coefficient of arresting rope PAB are related to damper settings. Therefore, this article mainly analyses how the stiffness and damping coefficient of arresting rope affects the maximum resistance. In order to avoid unreasonable set of parameters so that the range of motion of UAV during recovery is too large, the influence of the parameters on the maximum radius during recovery is also analysed.

Influence of the rope's stiffness $k_{l}$ on maximum resistance force $F_{\max }$ is researched. And when $c_{l}=40 \mathrm{~N} /\left(\mathrm{m} \cdot \mathrm{s}^{-1}\right)$, influence of the rope's stiffness $k_{l}$ on maximum overload and maximum radius is considered. The results are as Figure 11 and Figure 12:

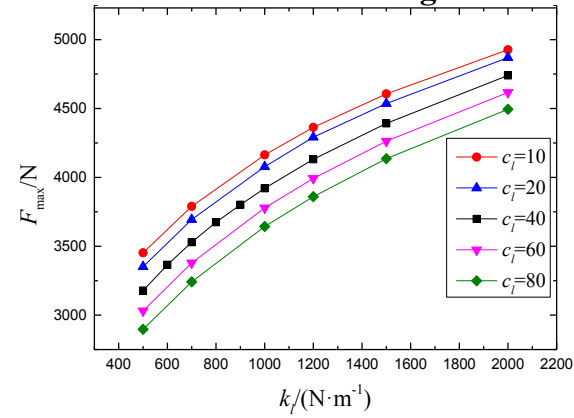

Figure 11. Influence of the rope's stiffness on maximum resistance force

The above two figures shows that the recovery performance with the changing of the stiffness of the arresting rope is consistent under different damping coefficients: the greater the stiffness is, the 
greater the maximum resistance and the maximum overload are, and the smaller maximum radius of the UAV's trajectory is. Besides, the slope of the curve is greater when stiffness is smaller.

Influence of the rope's damping coefficient $c_{l}$ on maximum resistance force $F_{\max }$ is studied. And when $k_{l}=500 \mathrm{~N} / \mathrm{m}$, influence of the rope's damping coefficient $c_{l}$ on maximum overload and maximum radius is considered. The results are as Figure 13 and Figure 14:

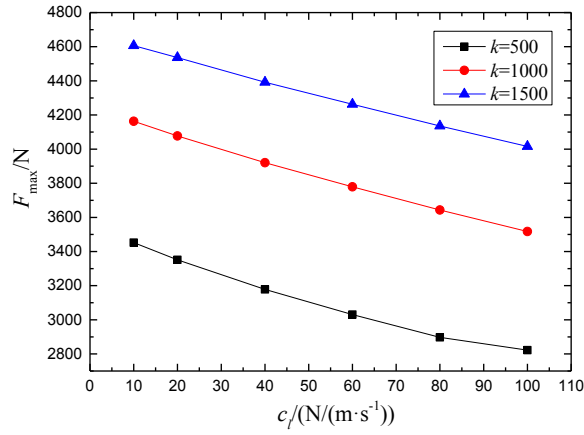

Figure 13. Influence of the rope's damping coefficient on maximum resistance force

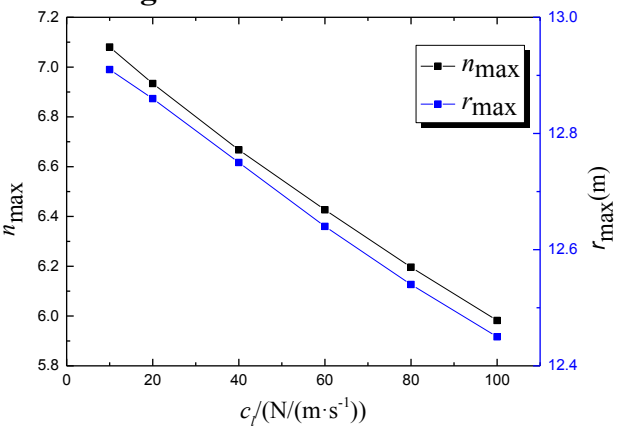

Figure 14. Influence of the rope's damping coefficient on maximum overload and radius

The above two figures shows that the recovery performance with the changing of the damping coefficient of the arresting rope is consistent under different stiffness: the greater the stiffness is, the lower the maximum resistance and the maximum overload are, and the smaller maximum radius of the UAV's trajectory is. And the trend is basically linear.

In addition, it can also be seen from the velocity curves of the aircraft during the recovery with different damping coefficients (as shown in Figure 15) that increasing the damping coefficient can reduce the peak velocity of each fluctuation and increase energy absorption. However, the influence mainly works in the initial stage of the recovery process, especially the change of the peak velocity of the first fluctuation after the collision.

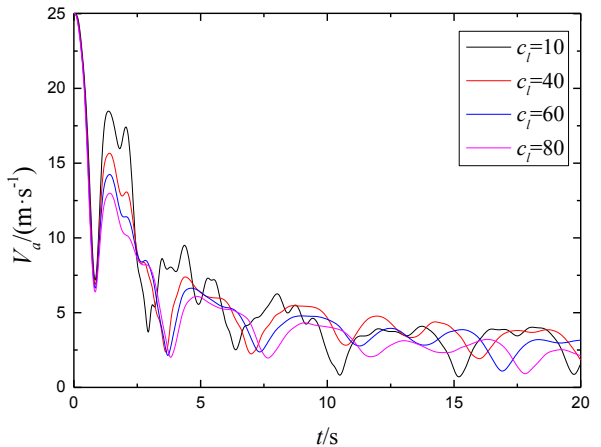

Figure 15. Velocity curves of UAV with different arresting rope's damping coefficient

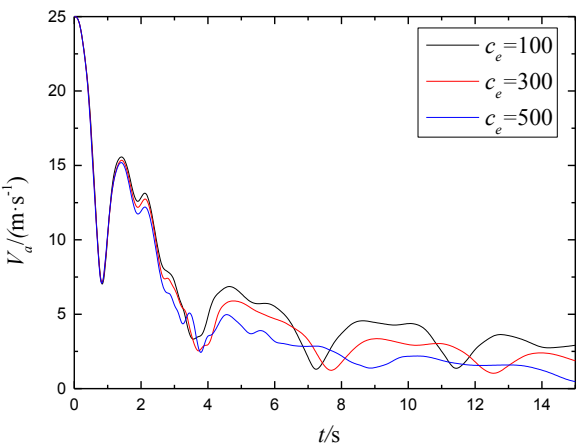

Figure 16. Velocity curves of UAV with different damping rope's damping coefficient

\subsection{Effect of the damping coefficient of damping rope}

The damping ropes EG and FG on the upper support of the recovery stand also contribute greatly to the cushioning and energy absorption effects of the recovery process. Therefore, its influence on recovery performance must also be considered. Different curves of velocity $V$ over time $t$ when damping coefficient of damping rope varies are compared as Figure 16.

It shows that the change of the damping coefficient of the damping ropes EG and FG has little effect on the initial buffering and energy absorption performance of the recovery process. There is no obvious difference of the peak velocity of the first fluctuation in curve, under the three different damping coefficients. However, as time goes on, increasing the damping coefficient of the damping rope can obviously improve the energy absorption effect. Using a larger damping coefficient, the fluctuation weakens, and the convergence tendency gets obviously faster. 
Compared with the former velocity curves when damping coefficient of the arresting rope PAB varies, as shown in Figure 15, it can be seen that the absorbing and buffering effect of the arresting rope $\mathrm{PAB}$ is mainly reflected in the initial stage of the recovery process, while the absorbing effect of the damping rope EG and FG responses later, mainly reflects in the convergence stage. The reason is that in the initial stage, deformation of arresting rope PAB caused by the aircraft is the main factor, the recovery stand scarcely rotates or deforms, and thus energy absorption mainly lies on the arresting rope; while the motion of UAV turns into rotating around the recovery stand, deformation of the arresting rope $\mathrm{PAB}$ reduces and the deformation of the damping rope $\mathrm{EG}$, FG caused by the rotation of the recovery stand $\mathrm{AD}$ becomes dominant energy absorption factor.

\section{Conclusions}

In this paper, a model of UAV vertical rope-type recovery system was established, and the UAV's position, attitude, velocity and resistance response curve during the recovery process were simulated. The effects of recovery speed of UAV, hit-rope position and stiffness as well as damping coefficient of arresting ropes on recovery performance were studied. These are the conclusions:

1) The effect of recovery velocity on the maximum overload is approximately linear and the lighter the UAV's weight is, the more sensitive the maximum overload is to the change of recovery velocity.

2) The maximum resistance force tends to decrease, when the hit-rope position is close to wing tip in wingspan direction, or close to the midpoint of recovery stand in height direction.

3) Increasing the stiffness of the arresting rope can reduce the maximum radius of the UAV's trajectory in the recovery process, but will increase the maximum overload. Increasing the damping coefficient can improve the buffering characteristics of the recovery process, which can reduce the maximum overload and the maximum radius of the UAV's trajectory during recovery.

4) Increasing the damping coefficient of the arresting rope PAB mainly improves the buffering and energy absorption performance in the initial stage of the recovery process, and reduces the maximum overload; while increasing the damping coefficient of the damping rope EG and FG can improve the concussion characteristics and speed up the convergence of UAV's velocity.

\section{References}

1. QIN B, WANG L. Summary of development of UAV. Winged Missiles Journal, 8:4-10 (2002).

2. ZHAO T. Development of shipborne UAV. Ship Electronic Engineering, 30(4):21-24 (2010).

3. HONG D, ZHOU L, ZHENG Z S. Research of the development of foreign small shipborne fixed-wing UAV recovery technology. Winged Missiles Journal, 4:50-54 (2014).

4. Dennis B D. Methods and apparatuses for capturing and recovering unmanned aircraft, including a cleat for capturing aircraft on a line: United States, 7059564B2.2006-06-13.

5. ZANG C X, CHU P J, HU Q, et al. The status and development trend of UAV recovery technology. Winged Missiles Journal, 3:39-42 (2016).

6. DAI C, ZHANG Y, XIA X Y, et al. Vertical rope-type recovery device of small UAV: China, 201761644U. 2011-03-16.

7. LU W, MA X P, ZHOU M, et al. Simulation analysis of dynamic characteristic of UAV ropehook recovery system. Acta Aeronautica et Astronautica Sinica. 36(10):3295-3304 (2015).

8. WANG H X, LIU C L, CHENG J. UAV recovery technology and its development. Winged Missiles Journal, 1:27-31 (2016).

9. B. Etkin. Dynamics of Atmospheric Flight (Beijing: Science press) pp 112-120 (1979).

10. FANG Z P, CHEN W C, ZHANG S G. Aeronautic Aircraft flight dynamics (Beijing: Beihang University Press) pp 174-180 (2005).

11. WANG Y L. Unmanned Aerial Vehicle Simulation in Flight envelope based on Matlab/Simulink. Nanjing: Nanjing University of Aeronautics and Astronautics, 2006:6-27. 